\title{
Type 2 cytokine-induced microRNA-29s suppressed both soluble ST2 release and IFNAR1 expression in human bronchial epithelial cells
}

\author{
Arisa Igarashi $^{1}$, Kenji Matsumoro ${ }^{1}$, and Akio Matsuda ${ }^{1}$ \\ ${ }^{1}$ National Research Institute for Child Health and Development
}

July 28, 2020

To the Editor:

Bronchial asthma is characterized by restricted airflow due to chronic airway inflammation, and frequent lower respiratory viral infections in early life are a significant risk factor for development of the disease. Previous studies demonstrated that anti-viral interferon (IFN) production, including of IFN- $\alpha$, IFN- $\beta$ and IFN- $\lambda$, by leukocytes and bronchial epithelial cells can be impaired in asthma patients. ${ }^{1}$ An epidemiological study found that allergic sensitization precedes wheeze during asthma development in children, suggesting that Type 2 (T2) conditions play a key role in the impaired anti-viral IFN production. Furthermore, a prospective cohort study showed that, regardless of the type of virus, each successive lower respiratory viral infection with wheeze increases the risk of asthma by about 1.5 fold. ${ }^{2}$ However, we still don't have a full understanding of the precise mechanism(s) of how respiratory viral infections under T2 conditions lead to development of asthma.

A meta-analysis of large-scale genome-wide association studies revealed that both $I L$ - 33 and its receptor, IL-33 receptor ( $I L-33 R$; also known as ST2 ), are closely associated with asthma development. ${ }^{3}$ Indeed, IL-33 expression was reportedly increased in rhinovirus-infected bronchial epithelial cells and correlated significantly with the disease severity of asthma. ${ }^{4}$ This suggests that virus-induced IL-33 in the airway may be fundamentally involved in the mechanistic links between viral infection and development and/or exacerbation of asthma. In addition, impairment of anti-viral IFN production was reported to cause necrosis - but not apoptosis - of the virus-infected epithelium, ${ }^{5}$ which results in release of bioactive IL-33.

MicroRNAs (miRNAs) are small, non-coding RNA molecules (containing about 22 nucleotides) that are found in diverse organisms. miRNAs regulate expression of a broad spectrum of target genes through RNA silencing and/or post-transcriptional regulation. Among them, microRNA-29a (miR-29a) was induced by respiratory syncytial virus (RSV) infection in a human lung adenocarcinoma cell line, A549, and suppressed expression of IFN $\left(\alpha, \beta\right.$ and $\omega$ ) receptor 1 (IFNAR1). ${ }^{6}$ Furthermore, miR-29a regulated the expression of soluble ST2 (sST2), a decoy receptor for IL-33, in human tenocytes. ${ }^{7}$ Based on those earlier findings, we focused on miR-29 in the present study. We hypothesized that T2 cytokine induces miR-29 expression in bronchial epithelial cells, leading to suppression of both sST2 release and IFNAR1 expression by epithelial cells, and culminating in asthma development and/or exacerbation.

Based on that hypothesis, we first examined whether T2 cytokine and inflammatory cytokine induced sST2 production in a human bronchial epithelial cell line, BEAS-2B. The detailed methods are described in Supporting Information. Specific ELISA showed that IL-4 and TNF- $\alpha$ synergistically induced sST2 release from BEAS-2B, in a dose-dependent manner (Figure 1A). Next, to examine the effects of miR-29 overexpression or inhibition on that cytokine-induced sST2 release, BEAS-2B cells were first transfected with miR-29 mimics or inhibitors for 24 hours and then stimulated with a combination of IL- 4 and TNF- $\alpha$ for 48 hours. 
The human miR-29 family consists of three mature members, i.e., miR-29a, miR-29b, and miR-29c. These miR-29s are encoded by the miR-29a/b-1 cluster on chromosome $7 \mathrm{q} 32.3$ and the miR-29c/b-2 cluster on chromosome 1q32.2, respectively (Figure 1B). ${ }^{8}$ The three family members share an identical seed sequence (Figure 1B), and their functional properties are thought to be similar. We examined the effects of miR-29a and miR-29b in this study. ELISA of the culture supernatants showed that inhibition of miR-29a or miR-29b significantly enhanced cytokine-induced sST2 release (Figure 1C). In contrast, overexpression of miR-29a or miR-29b almost completely inhibited that release, indicating that these miR-29s regulate sST2 release from bronchial epithelial cells under T2 conditions. Of note, neither inhibition nor overexpression of miR-29a or miR-29b had any effects on the protein levels of the ST2 receptor in the BEAS-2B cells (Figure 1D, upper panel). These results suggest that T2 cytokine-induced miR-29 plays a critical role in IL-33-dependent allergic inflammation through regulation of sST2 release from bronchial epithelial cells.

Furthermore, transfection of either miR-29a or miR-29b inhibitors significantly enhanced IFNAR1 protein expression in the BEAS-2B cells (Figure 1D, middle panel), which is consistent with earlier findings for miR-29a in A549 cells. ${ }^{6}$ Conversely, transfection of miR-29 mimics resulted in reduced IFNAR1 expression in BEAS-2B cells, suggesting that overproduction of miR-29s in bronchial epithelial cells may lead to suppression of antiviral responses by IFNs. Thus, we found that miR-29s simultaneously regulate the expression of both sST2 and IFNAR1 in bronchial epithelial cells. Our findings suggest the possibility that T2 cytokine-induced miR-29s in airway epithelial cells are key players in the development and/or exacerbation of asthma triggered by respiratory viral infections through both decreasing IFN-regulated antiviral activities and exacerbating IL-33-dependent allergic inflammation.

miRNAs are released from cells into the extracellular environment via exosomes, which can then fuse with target cells. This process can deliver various proteins and nucleic acids, including miRNAs, into even distant target/receiving cells. ${ }^{9}$ We, therefore, examined whether exosomes similarly export miR-29s from bronchial epithelial cells. BEAS-2B cells were stimulated with a combination of IL- 4 and TNF- $\alpha$ for 48 hours, and exosomal fractions were collected from the culture supernatants. Although qPCR detected both miR-29a and miR-29b in the exosomes even without that T2 cytokine stimulation (control), both of their copy numbers were significantly increased by that stimulation (Figure 2A). Furthermore, Western blot analysis also found that expression of CD81, an exosome marker, was enhanced by the cytokine stimulation (Figure 2B). These results suggest that T2 cytokine-stimulated epithelial cells release more exosomes containing more miR-29s than unstimulated cells.

This study has several limitations. First, no functional experiments were performed in this study to confirm the effects of the changes in sST2 release or IFNAR1 expression. In addition, we did not measure the expression levels of miR-29s in clinical samples.

Figure S1 summarizes our findings as a schematic illustration of bronchial epithelial cells. Based on those findings, we hypothesize that elevated nasal, bronchial and/or exosomal levels of miR-29s in infancy may be useful biomarker(s) for predicting later development of asthma, and further studies are needed. Our data suggest a new perspective that miRNAs are crucially involved in the association between viral infection and asthma development. We believe that our research has great significance in pointing to a novel direction for further studies and the existence of a new key player, i.e., miRNAs, in the relationship between viral infections and asthma development.

\section{Acknowledgments}

This work was supported in part by Grants-in-Aid for Young Scientists (B) from JSPS KAKENHI (15K19439 to A.I.) and grants from National Center for Child Health and Development (\#27-15 and \#2020B-4 to K.M.). We thank Mr. Lawrence W. Stiver (Quality Translation Co., Ltd.; Tokyo, Japan; qualityt@gol.com) for proofreading our English.

\section{Supporting Information}

Additional supporting information may be found online in the Supporting Information section at the end of 
the article.

\section{ORCID}

Akio Matsuda https://orcid.org/0000-0002-7781-0613

Kenji Matsumoto https://orcid.org/0000-0002-2630-6927

\section{Correspondence}

Akio Matsuda, Department of Allergy and Clinical Immunology, National Research Institute for Child Health and Development, 2-10-1 Okura, Setagaya-ku, 157-8535, Tokyo, Japan. Phone: +81-3-5494-7120 (ext. 4955); Fax: +81-3-5494-7173; E-mail: matsuda-a@ncchd.go.jp

\section{Authorship}

$\mathrm{AI}$ and AM designed the study, contributed to data collection and wrote the manuscript. KM interpreted the results and critically reviewed the manuscript. All authors read and approved the final manuscript.

\section{CONFLICTS OF INTEREST}

The authors have no conflicts of interest directly relevant to the contents of this article.

\section{FUNDING INFORMATION}

This work was supported in part by Grants-in-Aid for Young Scientists (B) from JSPS KAKENHI (15K19439 to A.I.) and grants from National Center for Child Health and Development (\#27-15 and \#2020B-4 to K.M.).

\section{REFERENCES}

1. Edwards MR, Strong K, Cameron A, et al. Viral infections in allergy and immunology: How allergic inflammation influences viral infections and illness. J Allergy Clin Immunol. 2017;140(4):909-920.

2. Bønnelykke K, Vissing NH, Sevelsted A, Johnston SL, Bisgaard H. Association between respiratory infections in early life and later asthma is independent of virus type. J Allergy Clin Immunol.2015;136(1):8186.

3. Torgerson DG, Ampleford EJ, Chiu GY, et al. Meta-analysis of genome-wide association studies of asthma in ethnically diverse North American. Nat Genet. 2011;43(9):887-892.

4. Jackson DJ, Makrinioti H, Rana BM, et al. IL-33-dependent type 2 inflammation during rhinovirus-induced asthma exacerbations in vivo.Am J Respir Crit Care Med. 2014;190(12):1373-1382.

5. Wark PA, Johnston SL, Bucchieri F, et al, Asthmatic bronchial epithelial cells have a deficient innate immune response to infection with rhinovirus. J Exp Med. 2005;201(6):937-947.

6. Zhang Y, Yang L, Wang H, Zhang G, Sun X. Respiratory syncytial virus non-structural protein 1 facilitates virus replication through miR-29a-mediated inhibition of interferon-alpha receptor. Biochem Biophys Res Commun. 2016;478(3):1436-1441.

7. Millar NL, Gilchrist DS, Akbar M, et al. MicroRNA29a regulates IL-33-mediated tissue remodelling in tendon disease. Nat Commun.2015;6:6774.

8. Kwon JJ, Factora TD, Dey S, Kota J. A Systematic Review of miR-29 in Cancer. Mol Ther Oncolytics. 2019;12:173-194.

9. Stoorvogel W. Functional transfer of microRNA by exosomes. Blood. 2012;119:646-648.

Figure legends

FIGURE 1 


\section{miR-29s suppress cytokine-induced expression of sST2 and IFNAR1 in BEAS-2B cells}

A, Cytokine-induced soluble ST2 (sST2) release from BEAS-2B cells. A human bronchial epithelial cell line, BEAS-2B cells, was stimulated with the indicated concentrations of IL- 4 and TNF- $\alpha$, alone or in combination, for $48 \mathrm{~h}$. The protein concentration of sST2 in each culture supernatant was measured by ELISA. B, Schematic representation of the gene structures of miR-29 family members. C, The effects of inhibition or overexpression of miR-29s on sST2 release from BEAS-2B cells were examined. Following 24-h transfection of $10 \mathrm{nM}$ of miRNA for miR-29 inhibitors, mimics or a negative control, the cells were stimulated with a combination of $100 \mathrm{ng} / \mathrm{ml}$ IL- 4 and TNF- $\alpha$ for $48 \mathrm{~h}$. The protein concentration of sST2 in each culture supernatant was measured by ELISA, and the data are shown as the mean $\pm \mathrm{SD}$ of triplicate samples. ${ }^{*} P<$ .05 by Student's $t$-test. D, Effects of inhibition or overexpression of miR-29s on the expression of ST2 and IFNAR1 proteins in BEAS-2B cells. BEAS-2B cells were treated as above in C. Whole-cell lysates were subjected to Western blot analysis of the expression of ST2, IFNAR1 and $\beta$-actin (as a loading control). The data are representative of two separate experiments. Chr.: chromosome; NC: negative control; IFNAR1: $\operatorname{IFN}(\alpha, \beta$ and $\omega)$ receptor 1 ; ACTB: $\beta$-actin.

\section{FIGURE 2}

\section{Epithelial-derived miR-29s in exosomes}

BEAS-2B cells were stimulated with a combination of $100 \mathrm{ng} / \mathrm{ml}$ IL- 4 and TNF- $\alpha$ for $48 \mathrm{~h}$. Cell culture supernatants were collected, and the exosomal fractions were extracted. A, The expression levels of miR-29a and miR-29b in the exosomal fractions were measured by qPCR. The data are shown as the mean \pm SD of triplicate samples. Statistical analysis was performed by Student's $t$-test. ${ }^{*} P<.05$. B, The expression of CD81, a marker of exosomes, was analyzed by Western blot analysis.

A

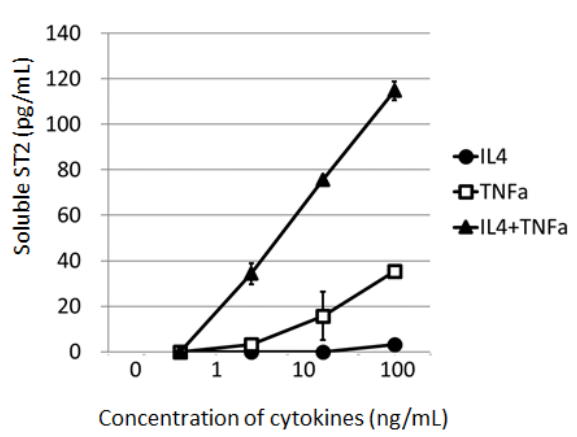

B

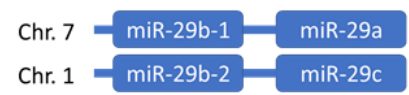

hsa-miR-29a-3p UAGCACCAUCUGAAAUCGGUUA hsa-miR-29b-3p \AGCACCAUUUGAAAUCAGUGUU hsa-miR-29c-3p UAGCACCAUUUGAAAUCGGUUA

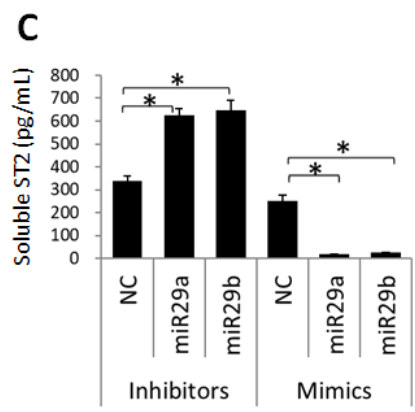

D

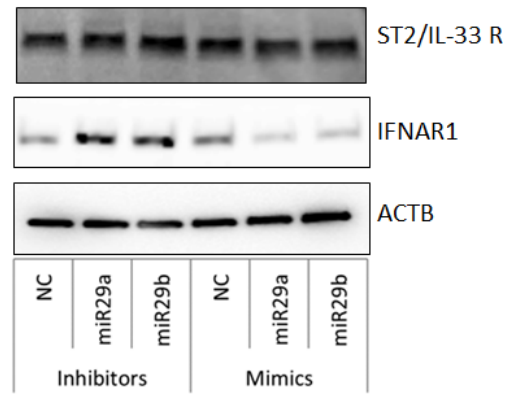


A

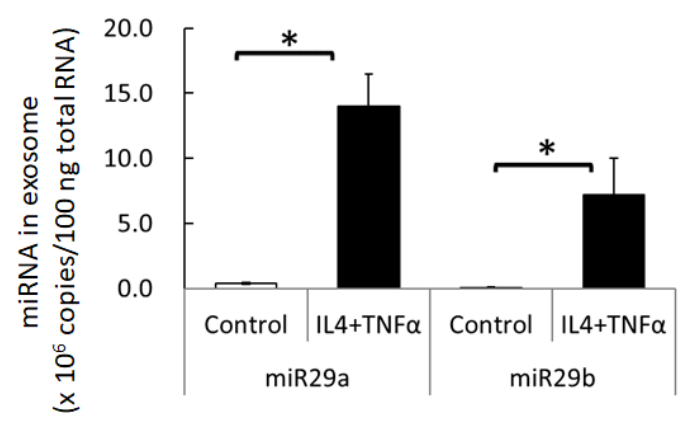

B

CD81

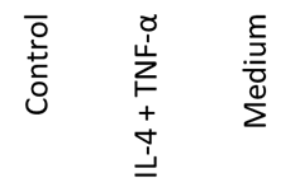

